\title{
A Comparison between the Efficacy of Bio-Oss, Hydroxyapatite Tricalcium Phosphate and Combination of Mesenchymal Stem Cells in Inducing Bone Regeneration
}

\author{
Surena Vahabi, DDS, MSc; Amirizadeh N, PhD; Shokrgozar MA², PhD; \\ Mofeed R, DDS, MSc; Mashhadi Abbas F, DDS, MSc; Aghaloo M³, DDS, MSc; \\ Sharifi $\mathrm{D}^{4}$, PhD; Jabbareh $\mathrm{L}^{5}$, DDS
}

Background: Recently, tissue engineering has been introduced as a regenerative treatment for bone defects. There is some evidence showing bone regeneration from mesenchymal stem cells (MSC) loaded on hydroxyapatite $\beta$-tricalcium phosphate (HA/TCP) as a scaffold in large defects. This study aimed to compare the quality and quantity of regenerated bone using Bio-Oss, HA/TCP and MSC loaded HA/TCP scaffolds.

Methods: Mesenchymal stem cells were aspirated from iliac crest bone marrow after extracting the first, second and third premolars and the first molar in five mature hybrid dogs. The cells were cultured and their osteogenic differentiation potential was evaluated after the third cell passage using Alizarin red staining in experimental conditions. The HA/TCP scaffold $(3 \times 3 \times 3 \mathrm{~mm})$ was loaded with undifferentiated mesenchymal stem cells. Bilateral bone defects were then prepared in the jaws using trephine burs. The defects were randomly filled with $\mathrm{HA} / \mathrm{TCP}$, Bio-Oss, or HA/TCP + MSCs. One defect served as a control and was left as an empty cavity. All defects except the control defect were covered with an absorbable membrane. Histological and histomorphometric evaluations were conducted after 6 weeks and data were subjected to analysis of variance (ANOVA) $(p<0.05)$.

Results: The empty cavity demonstrated more bone formation $(60.80 \%)$ than the HA/TCP $(44.93 \%)$ and Bio-Oss $(40.60 \%)(p<0.05)$ groups. However, the difference from the HA/TCP + MSCs group was not significant $(46.38 \%)$ $(p>0.05)$.

Conclusion: An MSC-loaded HA/TCP scaffold is a more effective alternative than BioOSS or HA/TCP in inducing bone regeneration.

(Chang Gung Med J 2012;35:28-37)

Key words: mesenchymal stem cells, regeneration, alveolar bone, hydroxyapatite, tricalcium phosphate

\footnotetext{
From the Dental School, Shahid Beheshti Medical University, Tehran, Iran; 'Iranian Blood Transfusion Organization, Tehran, Iran; ${ }^{2}$ Pasteur Institute, Tehran, Iran; ${ }^{3}$ Dental School, Qazvin Medical University, Tehran, Iran; ${ }^{4}$ Veterinary School, Tehran Medical University, Tehran, Iran; ${ }^{5}$ Dental School, Mashhad Medical University, Iran.

Received: Jan. 31, 2011; Accepted: Sep. 21, 2011

Correspondence to: Dr. Surena Vahabi, Dental School, Shahid Beheshti Medical University, Tehran, Iran. Periodontics unit, Dental School, Shahid Beheshti Medical University, Daneshjoo BLVD, Velenjak, Tehran, Iran. Tel: 98-21-29902314;

Fax: 98-21-88560920; E-mail: ivsure1@ gmail.com
} 
$\mathrm{T}$ he first six months post tooth extraction is concomitant with a modeling/remodeling phase which results in up to $40 \%$ and $60 \%$ reduction in bone height and width respectively. ${ }^{(1)}$ An inadequate bone level in anterior regions is a common problem which compromises the success of implants in restoring the function and esthetics of a lost tooth. ${ }^{(2)}$ Small and moderate size defects ( $\leq 3 \mathrm{~mm}$ and 4-6 $\mathrm{mm}$, respectively) can be effectively treated using a combination of soft tissue grafting and guided bone regeneration (GBR) techniques. GBR is defined as a surgical procedure that utilizes barrier membranes to direct the growth of new bone at sites having insufficient volumes or dimensions of bone for proper function for implant placement. Large defects, over 7 $\mathrm{mm}$, however, are more complicated and cannot be effectively managed. ${ }^{(3)}$ Currently the gold standard method in managing large alveolar ridge defects is an autogenous bone graft. ${ }^{(4,5)}$

Although alveolar defects may be reconstructed using mandibular augmentation techniques via autogenous bone grafts, this is considered a major procedure involving a bone graft technique which causes deformity in the donor site. Furthermore, the unknown rate of bone absorption and the necessity to allow several months for the site of the implant to heal, make grafting a sensitive treatment option. ${ }^{(6)}$ Considering the difficulties associated with the use of autogenous bone grafts, it is necessary to propose a new technique to handle bone defects.

Nowadays, researchers have turned to tissue engineering techniques as an alternative method in regenerating jaw defects. The efficacy and success rate of different techniques and materials in different regions of the jaw have been evaluated. However, there is little information on the combined efficacy of synthetic hydroxyapatite $\beta$-tricalcium phosphate (HA/TCP) scaffolds and mesenchymal stem cells (MSCs) in regenerating large alveolar ridge defects.

Vacanti et al. were the pioneers who proposed tissue engineering as a new method, which involved regenerating new tissue from isolated cells loaded on biocompatible scaffolds in the presence of growth factors. ${ }^{(7)}$

MSCs are capable of multiple proliferations without losing their osteogenic properties. The proliferative and osteogenic characteristic of MSCs makes them suitable candidates for reconstructing bone defects in clinical conditions. ${ }^{(8)}$ The pathogno- monic characteristic of these cells is their ability to differentiate into different types of mesoderm cells such as chondrocytes, myocytes, tonocytes and osteoblasts. ${ }^{(9)}$ Mesenchymal stem cells can be isolated from bone marrow, periosteum, trabecular bone, adipose tissue, synovium, skeletal muscles and deciduous teeth. ${ }^{(10)}$ Bone marrow is a common source of MSCs. These cells can easily be aspirated from bone marrow in minimal amounts, cultured and preserved in laboratory conditions.

Yoshikawa et al. (1996) reported that hydroxyapatite-loaded mesenchymal stem cells are comparable to autogenous cancellous bone particles and bone marrow particles. ${ }^{(11)}$ Boo et al. revealed similar osteogenic potential in beta tricalcium phosphate (a soluble substance) and hydroxyapatite ${ }^{(12)}$ for clinical purposes. This study aimed to evaluate the process of remodeling and bone development to reconstruct mandibular defects for succeeding implants.

\section{METHODS}

This experimental study was performed on 5 hybrid dogs. The sample size was determined with respect to previous studies, pilot evaluations and matching of confounding variables, and was confirmed by a statistician. The study was approved by the Institutional Animal Care and Use Committee of Shahid Beheshti Medical University. Data forms and charts were completed for each sample after histological evaluations.

\section{Extraction of mandibular teeth, isolation of stem cells and cell culture}

Five male hybrid dogs with identical ages of 1 year and mean weights of $25-35 \mathrm{~kg}$ without any specific health conditions were considered for this study. The dogs were kept in the laboratory for one week for adaptation, vaccination and anti-parasite therapy. After performing general anesthesia on the dogs using an intramuscular injection of xylazine hydrochloride $2 \%(0.1 \mathrm{mg} / \mathrm{kg})$, their mouths were rinsed with chlorhexidine $0.2 \%$. The first, second and third premolars and the first molars were cut into halves using cylindrical burs and then extracted (Fig. 1A, B). Bone marrow 10-20 cc was then aspirated from the animals' iliac crest and transferred to the stem cell laboratory of the Institutes of Blood Transfusion in $50 \mathrm{cc}$ containers with 7500 units of 
heparin.

The cells were cultured in Dulbecco's Modified Eagle's Medium (DMEM) (Life Technology, Gibco, Grand Island, NY, USA), fetal bovine serum (FBS) 15\% (Life Technology, Gibco) and $100 \mu \mathrm{L}$ penicillin/streptomycin (Life Technology, Gibco),-in sixblock containers for two days. Non-adhering cells were excluded from the culture plates. The adhering cells were further expanded under experimental conditions at $5 \% \mathrm{CO}_{2}$ and $37^{\circ} \mathrm{C}$ and their culture plate was renewed every three days.

\section{In vitro osteogenic differentiating potential of cells}

After the third cell passage, culture plates were removed and the osteogenic environment containing $0.2 \mathrm{mM}$ ascorbic acid (Sigma, St. Louis, MO, U.S.A.), $10^{-8}$ dexamethasone (Sigma) and $\mathrm{Na}-\beta$ glycerophosphate (Sigma) was added to the isolated bone marrow cells. Cells were subsequently cultured in the new environment for 7 weeks. The culture plates were renewed three times a week. After seven weeks, the cells were fixed with formalin solution $10 \%$ and the osteogenic matrix was ultimately stained with alizarin red (Sigma) for 15 minutes at room temperature and prepared for evaluation.

\section{Implant preparation}

Synthetic hybrid biphasic scaffolds (Kasios, Lanauguet, France) available in a 40/60 ratio with dimensions of $3 \times 3 \times 3 \mu \mathrm{m}$ were obtained. These structures contained HA (60\%) and $\beta$-TCP (40\%) with 300-500 mm porosities (HA/TCP Kasios). Scaffolds were initially covered with bovine type I collagen (Sigma) 24 hours prior to loading with stem cells. The scaffolds were then immersed for 2 hours at $37^{\circ} \mathrm{C}$ in a $0.2 \mathrm{cc}$ DMEM environment containing 5 X $10^{5}$ mesenchymal stem cells, all of which had undergone the third cell passage and were allowed to precipitate on the scaffold.

\section{Decalcification of scaffolds of bony specimens and microscopic evaluation using electron and light microscopy}

Specimens taken previously from the dog jaws during surgery were fixed in formalin solution $10 \%$ and PBS for 12 hours and placed in increasing concentrations of isopropanol and subsequently in paraffin. The same specimens after that have been put in the jaws of the dogs after healing of the defects. Three sections with 5- $\mu \mathrm{m}$ thickness were prepared from each specimen; these were observed under a light microscope (x 100).

To prepare the samples for scanning electron microscopy (SEM) analysis, after 24 hours MSCloaded HA/TCP samples were fixed with $2 \%$ paraformaldehyde and glutaraldhyde and placed in increasing concentrations of alcohol and examined with by SEM. SEM images were obtained and digitally recorded using a Jeol 660 instrument (Jeol Inc., Peabody, MA, U.S.A.).

\section{Implantation of scaffolds in dogs' mandibles}

Four weeks after tooth extraction and complete healing of the edentulous ridge, the MSC-loaded HA/TCP scaffolds were transferred to the animal surgery center of the veterinary school of Tehran University. The dogs were anesthetized as described and their mouths were rinsed with $0.2 \%$ chlorhexidine. After bilateral local anesthesia of the jaw with lidocaine, a crestal incision was performed and the mucoperiostal flap was retracted to expose the bone. Four cavities ( $10 \times 10 \times 10 \mathrm{~mm})$ were drilled on each side of the jaw using a trephine bur with a tip diameter of $10 \mathrm{~mm}$ (Fig. 1C). The cavities were prepared with $5 \mathrm{~mm}$ interspaces, the first of which was prepared at $5 \mathrm{~mm}$ from the reference point (anterior remaining tooth). Biocollagen membrane (BCG, Bovine-drived collagen, Bioteck, Arcugnano Vicenza, Italy) was applied to cover the cavities except for the empty cavity and were identically fixed by sutures in all groups (Fig. 1D). The cavities were randomly allocated into the following groups.

A: Scaffold HA/TCP + MSCs + BCG (8 scaffold particles were placed in each cavity)

B: Scaffold HA/TCP + BCG (8 scaffold particles were placed in each cavity)

C: Bio-Oss (Geistlich Biomaterials, Wollhusen, Switzerland) + BCG

\section{D: Empty cavity}

The incisions were then closed with absorbable suturing materials. The dogs were subjected to postoperative prophylactic antibiotic therapy with cefazoline $(22 \mathrm{mg} / \mathrm{kg})$ for 1 week and their mouths were rinsed with $0.2 \%$ chlorhexidine solution two times a week. In order to reduce pain and postoperative discomfort, they were also prescribed analgesics. Each dog was given a code and their characteristics were 

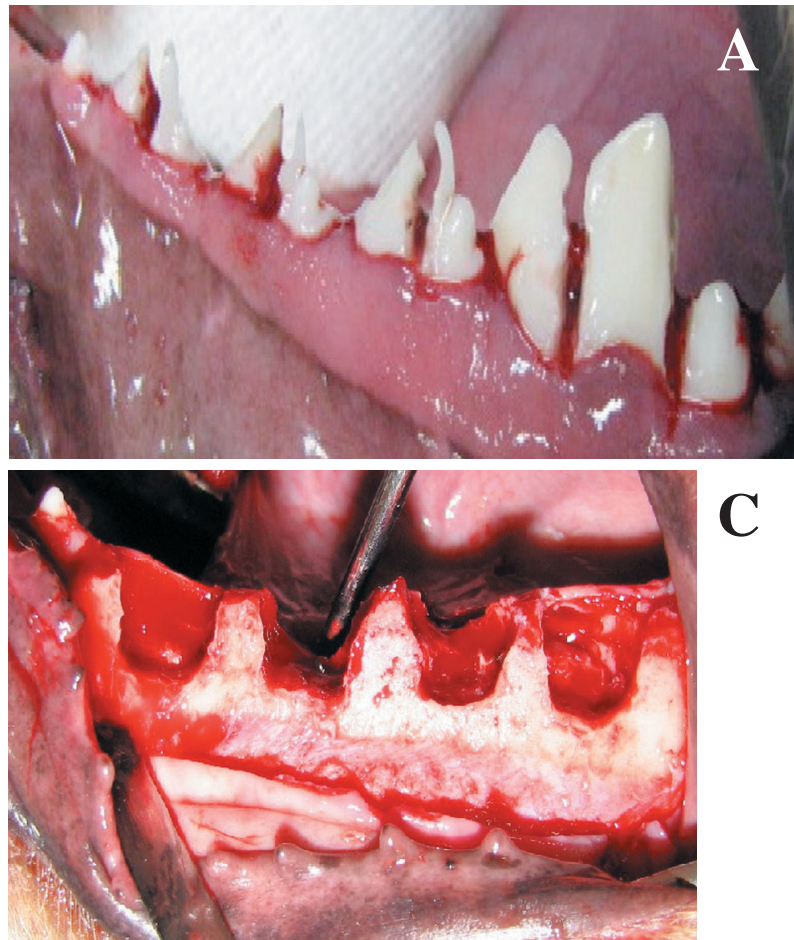
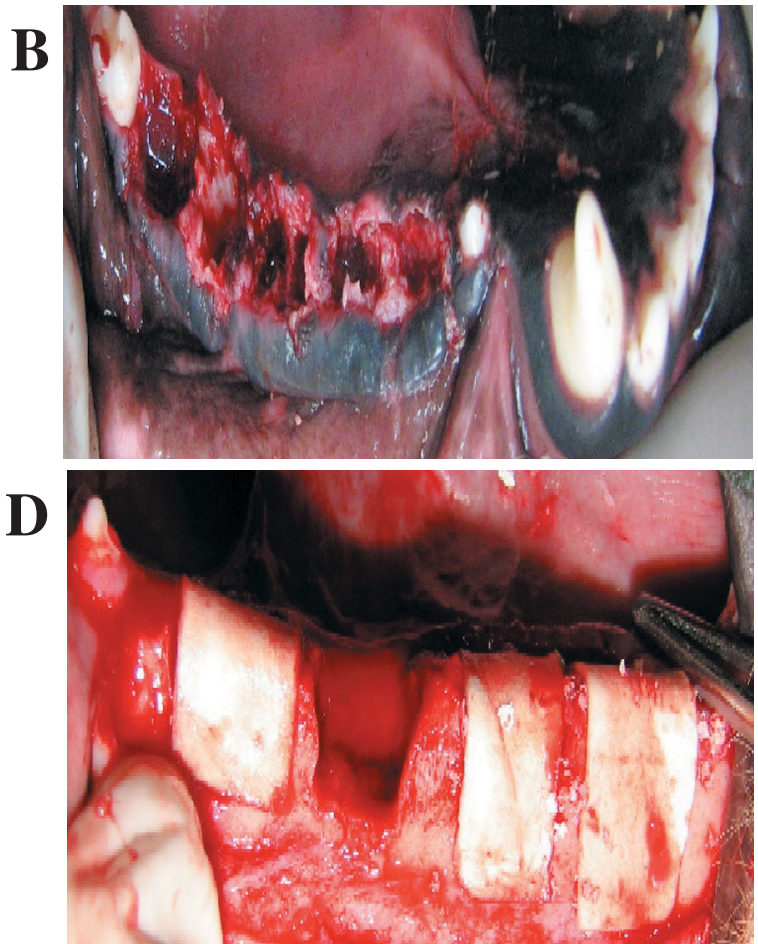

Fig. 1 (A) The teeth are sectioned with an air motor prior to extraction (B) Site of the extracted teeth (C) Cavities are drilled with a trephine bur. (D) The cavities are filled with bone substitutes and covered with absorbable membranes.

recorded on the respective forms. All of them received identical diets of soft food.

\section{Preparation of the samples for hematoxylin and eosin (H \& E) staining and light microscopy evaluation}

Eight weeks after the graft surgery, the dogs were sacrificed via the vital perfusion technique and samples were obtained using the block section technique, kept in $10 \%$ formalin solution and transferred to the department of Oral and Maxillofacial Pathology, Shahid Beheshti University of Medical Sciences, Tehran, Iran. Bone samples were kept in $10 \%$ formic acid solution for four weeks, cut into paraffin blocks of $5 \mu \mathrm{m}$ thickness and prepared for histologic evaluation. The blocks were stained with $\mathrm{H} \& \mathrm{E}$-and the lamellas were prepared for qualitative evaluation of bone or organic matrix formation, presence of scaffold, acute or chronic inflammatory cell infiltration or the severity of inflammation evaluated by the presence of giant cells and osteoclasts under a light microscope (Nikon Eclipse E400, Nikon, Samida KU, Japan). The microscopic images of each defect at a the magnification $\times 40$ were transferred to a computer using a digital camera (Nikon Fuji HC300 Nikon) and were processed by Photoshop histophotometry software using different color values and subtractions to calculate the percentage of woven bone or newly formed bone by histomorphometric evaluation. To reduce potential bias, the images were analyzed according to their given codes. Areas of newly formed bone, residual materials and soft tissue were marked manually with a computer mouse and calculated by computer.

\section{Statistical analysis}

Data were submitted to SPSS ver. 12 (IBM Corporation, NY, U.S.A.) for analysis. For qualitative data, simple and relative frequency was reported whereas for quantitative data, mean and SD were calculated. The univariate Kolmogorov Smirnov test was utilized to determine the normal distribution of data. Comparison of quantitative data within groups was performed using analysis of variance (ANOVA) followed by a post hoc Tukey's test when ANOVA suggested a significant difference between groups 
$(p<0.05)$.

\section{RESULTS}

\section{Cell cultures}

In the primary cell cultures, mononucleated dog bone marrow cells, fibroblast-like cells and a few round cells were detected. The number of round cells decreased after cell passages and the number of

\section{A}

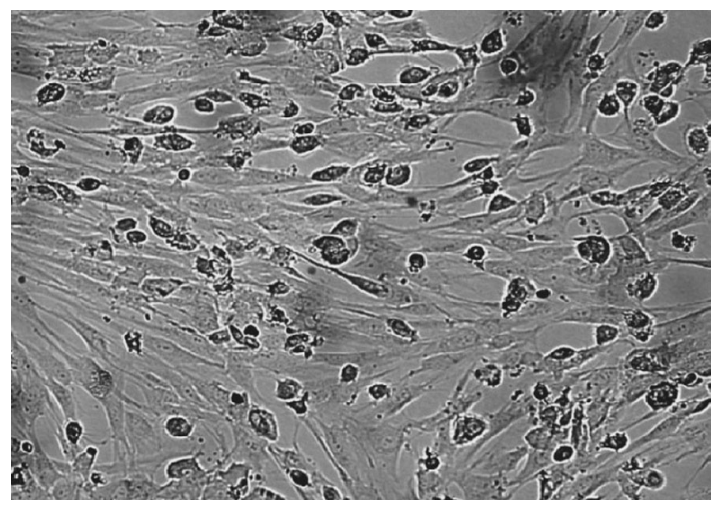

B

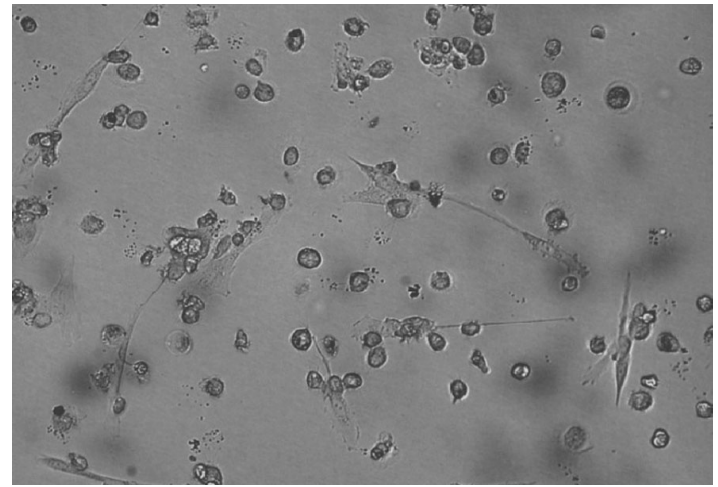

C

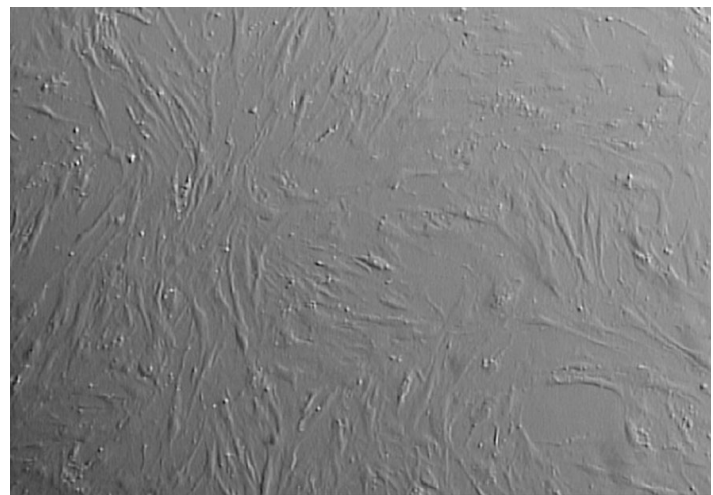

Fig. 2 A to $\mathrm{C}$ illustrate a decrease in the number of round cells attached to culture plates and an increase in the number of fibroblast-like mesenchymal stem cells in numerous cell passages (Contrast phase microscope). fibroblast-like mesenchymal stem cells increased (Fig. 2).

\section{Osteoblastic differentiation-alizarin red stain- ing}

In the osteogenic induction environment, cells were parted from the culture plates and floated in the culture environment. Nodular structures were observed in some parts of the culture plates. Mineralized tissues absorbed alizarin red stains and were spotted as red areas (Fig. 3).

\section{Cell penetration into HA/TCP scaffold}

Light microscopic images of cells containing HA/TCP scaffolds revealed proliferation and penetration of MSCs in the scaffold (Fig. 4).

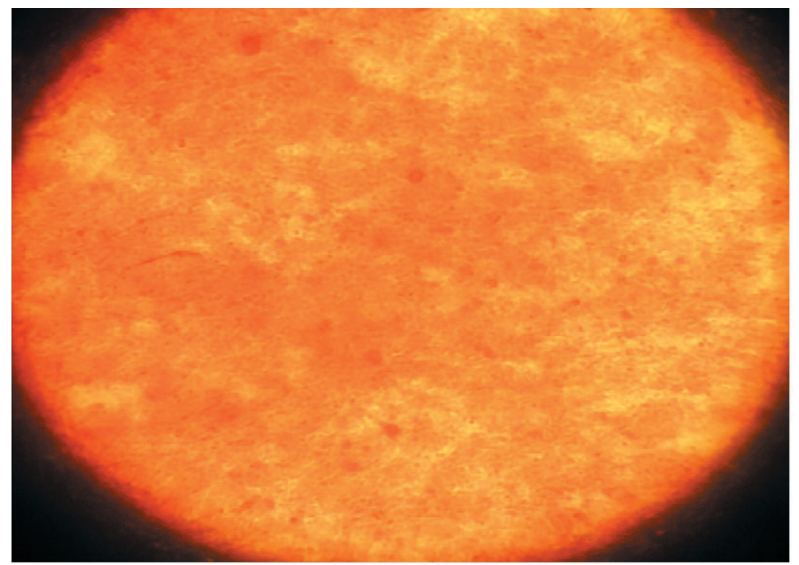

Fig. 3 Alizarin red staining. The arrow shows in vitro mineralized osseous tissues (Contrast phase microscope).

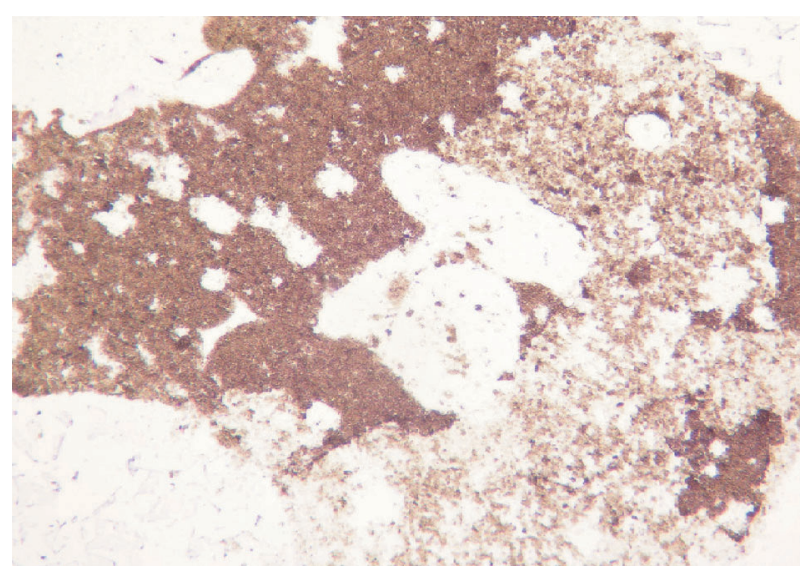

Fig. 4 Light microscopic view of (arrow) MSC-containing decalcified scaffolds (magnification $x$ 100). 
Electron microscope images revealed connections between the superficial and deep surfaces of the scaffolds. Mesenchymal stem cells attached to the collagen fibers of the surface of the scaffolds had a diverse morphology. The presence of these cells in the deep layers of the scaffold further confirmed their penetration into the scaffold (Fig. 5).

\section{Histological evaluations}

One dog was excluded because of technical problems and thus four dogs with 30 defects (cavities) were considered for histological and histomorphometric evaluations. Light microscopic views showed healing of the defects with the formation of different degrees of trabecular and woven bone and bone marrow spaces. The scaffold + MSC group was more likely to form dense mineralized tissue with less bone marrow space (Fig. 6).

Table 1 demonstrates the mean percentage of woven bone in the experimental groups. The Scaffold + MSC group displayed the highest percentage of woven bone $(47.5 \%)$ and the Bio-Oss and scaffold groups showed the least amount of woven bone (both $31.3 \%$ ), however the difference was insignificant $(p>0.05)$.

Cuboidal osteoblastic cells were observed in all groups. Osteocytes were also found in the lacunae in the newly formed bony trabeculae. Compared with the other groups, the Scaffold + MSC group showed more cells with better quality. None of the specimens showed evidence of severe inflammation or foreign body reaction and only one sample from the Bio-Oss and empty cavity groups showed the presence of

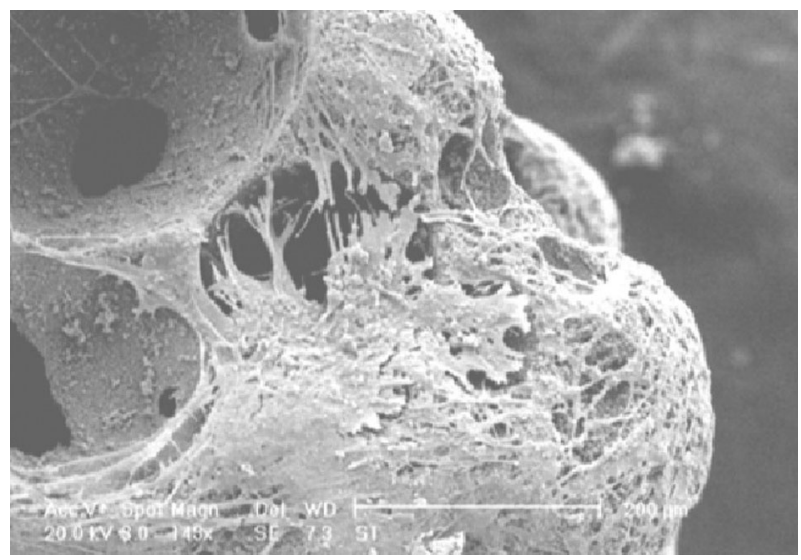

Fig. 5 Relatively normal distribution of MSCs on the HA/TCP scaffolds (high magnification 1/100)

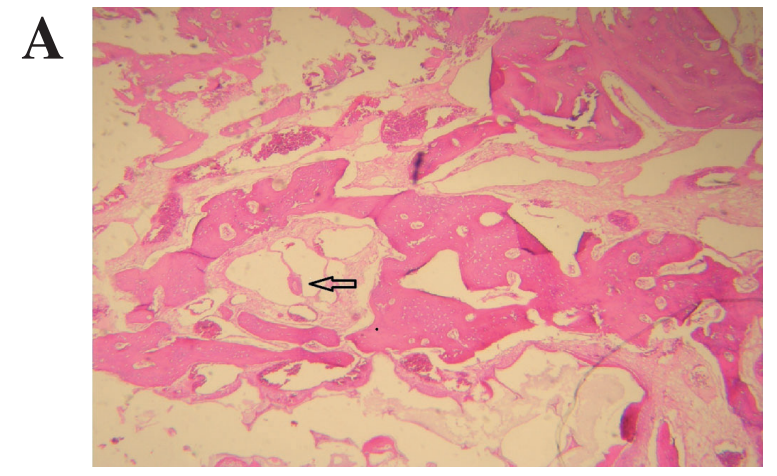

B
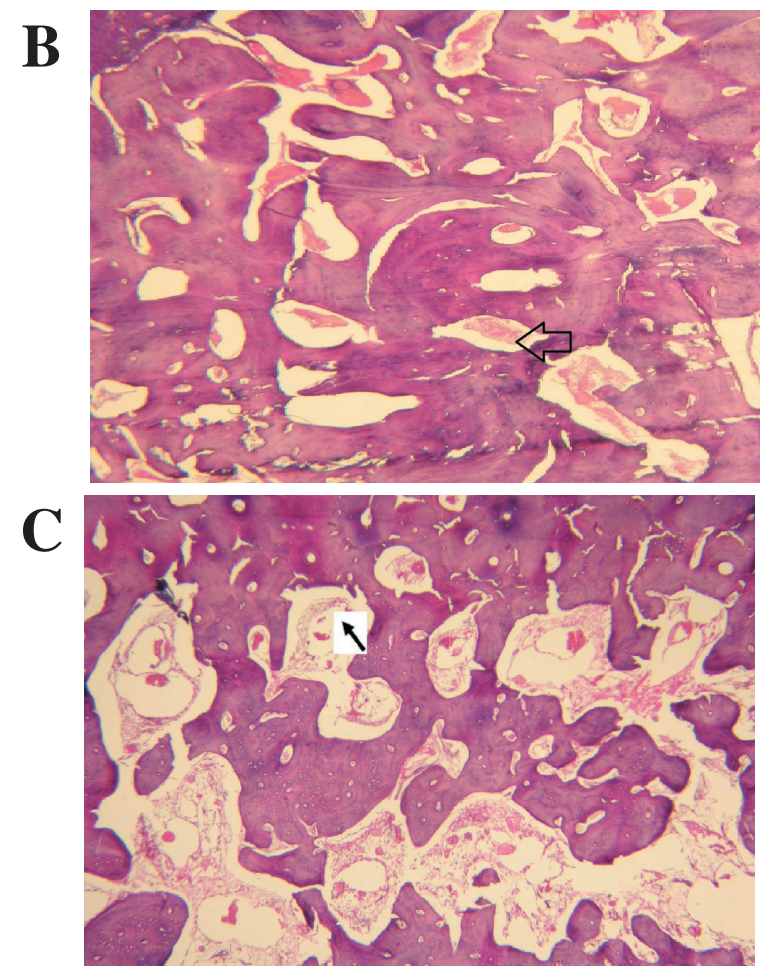

D

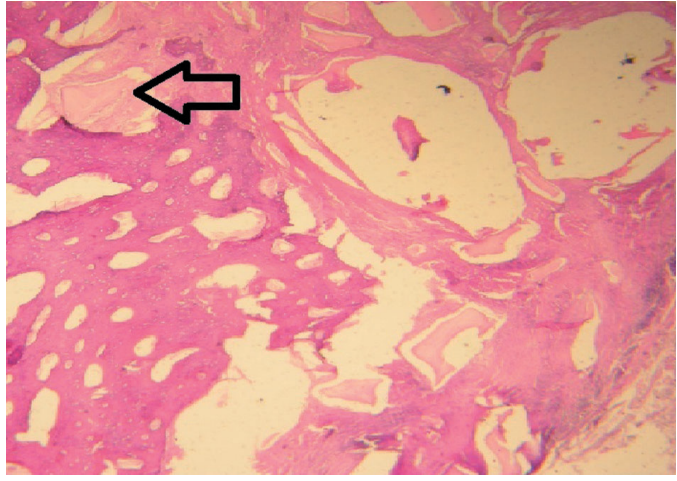

Fig. 6 Bone formation and remnant particles (arrow) in the bony defects in (A) the Scaffold + MSC group, (B) Scaffold group, (C) Bio-Oss group, and (D) empty cavity group (hematoxylin and eosin, magnification $x 40$ ). 
Table 1. Percentage of Woven Bone Observed under Light Microscopy and Histomorphometric Data on the Percentage of Bone Fill and Remnant Particles in Experimental Groups

\begin{tabular}{cccc}
\hline Group & Mean woven bone \pm SD & Mean bone fill \pm SD & Mean remnant particles \pm SD \\
\hline Scaffold + MSC & $47.5 \pm 20.5 \%$ & $46.38 \pm 13.49 \%$ & $14.62 \pm 10.35 \%$ \\
Bio-Oss & $31.3 \pm 14.6 \%$ & $40.60 \pm 9.15 \%$ & $16.46 \pm 8.87 \%$ \\
Scaffold & $31.3 \pm 13.6 \%$ & $44.93 \pm 8.81 \%$ & $14.87 \pm 8.35 \%$ \\
Empty cavity & $36.7 \pm 15.1 \%$ & $60.80 \pm 9.07 \%$ & - \\
\hline
\end{tabular}

giant cells. In the interventional groups, remnants of $\mathrm{HA} / \mathrm{TCP}$ and Bio-Oss were in contact with newly formed bone (direct bone contact).

The percentages of the newly formed bone in the cavities (trabecular or woven bone) were calculated histomorphometerically. The mean bone fill and remnant particles in the four groups are reported in Table 1. Bio-Oss had the most remnant particles $(16.46 \%)$ and the Scaffold + MSC group the least $(14.62 \%)$, but the difference was insignificant in the three interventional groups $(p>0.05)$.

Pair wise comparisons of bone fill between groups are provided in Table 2 . The empty cavity displayed the highest percentage of bone fill $(60.8 \%)$ and Bio-Oss showed the lowest (40.6\%). The Scaffold + MSC group had the second highest bone fill $(46.38 \%)$ with no significant difference compared with the empty cavity group $(p>0.05)$. The Scaffold and Bio-Oss groups displayed significantly less bone fill compared with the empty cavity $(p<0.05)$. The

Table 2. Pair Wise Comparisons of Bone Fill in Groups, and Histomorphometric Evaluation

\begin{tabular}{lcc}
\hline Groups & Mean difference \pm SD & $p$-value \\
\hline Scaffold + MSC Vs. Bio-Oss & $5.79 \pm 5.20$ & 0.685 \\
Scaffold + MSC Vs. Scaffold & $1.46 \pm 5.20$ & 0.992 \\
Scaffold + MSC Vs. & $14.42 \pm 5.62$ & 0.073 \\
$\quad$ Empty cavity & & \\
Bio-Oss Vs. scaffold & $4.33 \pm 5.20$ & 0.839 \\
Bio-Oss Vs. empty cavity & $20.20 \pm 5.62$ & 0.007 \\
Scaffold Vs. empty cavity & $15.87 \pm 5.62$ & 0.042 \\
\hline
\end{tabular}

three interventional groups failed to display significant bone fill $(p>0.05)$.

\section{DISCUSSION}

The findings of the present study revealed that the empty cavity had significantly greater bone formation capacity compared with the Bio-Oss and Scaffold groups $(p>0.05)$ with insignificant differences compared with the Scaffold + MSC group $(p>$ $0.05)$. The Scaffold + MSC group displayed a greater percentage of bone formation with less foreign body reaction whereas the Bio-Oss group displayed the lowest percentage of bone formation with more foreign body reaction among the 3 interventional groups. However, the differences were insignificant $(p>0.05)$. Our comprehensive search of the literature failed to retrieve any identical study comparable with ours in terms of design and bone defects; however, other similar sources of evidence are discussed below.

In a similar study by Jafarian et al. (2008) $\mathrm{HA} / \mathrm{TCP}+\mathrm{MSC}$ displayed greater bone formation capacity compared with Bio-Oss + MSC and HA/TCP alone; however, the difference between the cell loaded HA/TCP group and cell loaded Bio-Oss group was not significant. ${ }^{(13)}$ The results of the present study were in agreement with that of Jafarian; however, there were slight methodological differences in that they failed to include an empty cavity as a control and moreover, the lesions in the latter study were prepared through and through in the inferior border of the ridge. These lesions were "contained", giving them greater chance of bone formation; however, the cavities lacked buccal and lingual walls in this study.

In a light microscopic evaluation performed by 
Ito et al. (2005), mature bone formation occurred more rapidly in dog MSCs and the platelet-rich-plasma (PRP) group compared with the autogenous bone particle group, Bio-Oss group and empty cavity group. The lowest amount of bone formation occurred in the Bio-Oss group, which was in agreement with our findings in the present study. They reported that Bio-Oss particles were evident in the fibrous tissue without any evidence of bony mineralization. Furthermore, dog MSC/PRP displayed more bone formation compared with the empty cavity. ${ }^{(14)}$ The scaffold used in the latter study was PRP, which is rapidly absorbed and replaced by bony tissue. Yet, this substance needs to be further evaluated in terms of absorption and ability to preserve the defect until there is complete bone formation. In cases of rapid absorption, PRP would not yield different results compared with an empty cavity.

Bone substitutes should be gradually absorbed and replaced by vital bone tissue. The absorption rate of Bio-Oss is not fully known and most studies have demonstrated that a limited amount of bone slowly replaces Bio-Oss. ${ }^{(15-17)}$ Since the degree of mineralization is based on subjective evaluations under a light microscope, the controversial results may be attributed to the different perceptions of the raters.

The manufacturers of the HA/TCP scaffolds used in the present study (Kasios) claim that bone formation and superficial absorption of the scaffold occur 6 and 12 weeks, after placement, respectively.

The 6-week duration of this study was based on a pilot test to evaluate the quantity and quality of newly formed bone in a minimum period of time. Hence, the scaffold and Bio-Oss were not completely absorbed and were regarded as foreign bodies in the interventional groups. The empty cavity, however, underwent a normal healing process, showed no signs of foreign bodies, and had the greatest percentage of bone formation in histomorphometric evaluation. A suitable scaffold is one that is absorbed at a reasonable interval from bone formation. ${ }^{(14)}$ Nevertheless, further evaluations are required to find a scaffold that does not intervene with the normal ossification process. The finding in the present study revealed that the HA/TCP scaffold was absorbed more rapidly and induced more mineralization in the $\mathrm{HA} / \mathrm{TCP}+\mathrm{MSC}$ group than the scaffold group and Bio-Oss group alone.

It is still unclear why clinicians turn to bone substitutes, despite the greater percentage of physiologic bone formation by the empty cavity. The macroscopic view of the dogs' ridges revealed unevenly dipped sites over the empty cavity areas, whereas other sites treated with bone substitutes were at an even height compared with the other parts of the alveolar ridge. Dipped areas in the ridge compromise the aesthetic demands of patients in anterior regions and marks the necessity of using scaffolds in a defective alveolar ridge prior to implant placement.

The cavities drilled on dogs' alveolar ridges in the present study were similar to those of Ito et al. They also observed that in the $4^{\text {th }}$ week after placing the bone substitutes, the newly formed bone in the dog MSC/PRP group extended to the alveolar ridge margins while bone formation occurred incompletely in other groups and, in particular, the empty cavity group. ${ }^{(14)}$ Our study was limited in that we could not calculate the bone formation in proportion to the total defect area (meaning from the top of the ridge to the base of the defect area).

The cells in this study were loaded on the scaffolds by means of bovine collagen type $\mathrm{I}^{(13,17,18)}$ Numerous studies have assessed the effect of collagen type I on bone formation. Niemeyer et al. reported that when dog MSCs are loaded on collagenous scaffolds covered with hydroxyapatite, more ossification genetic markers are found than when MSCs are loaded on TCP scaffolds; this may occur because this matrix imitates normal bone matrix. ${ }^{(19)}$ Yang et al and Weinord et al also demonstrated the positive effects of collagen type I on attachment, proliferation, differentiation and bone formation of isolated mesenchymal stem cells from bone marrow on hydroxyapatite and TCP scaffolds. ${ }^{(21,20)}$ In the present study, all scaffolds in MSC loaded or cell free groups were immersed in bovine collagen type I for 24 hours before being placed in the cavities. This was identically performed for all groups to calibrate the effect of collagen on bone formation in the two groups.

The light microscopic evaluation revealed that the bone formed in the Scaffold + MSC group was more compact with less bone marrow spaces as well as more osteocytes in the lacunae than the other groups. In Jafarian's study, the cell loaded scaffold group also displayed the most osteoblast- like cells which is probably because of differentiation of MSCs into osteoblasts. ${ }^{(13)}$ It would have been better if 
we had attempted to mark the MSCs through their differentiation process into osteoblasts. Moreover, the MSCs in Jafarian's study were isolated from radius bone marrow, so there may be a significant tendency toward forming compact bone associated with the origin of the cells. Wu et al (2009) loaded the TCP scaffolds with osteoblasts after isolating bone marrow stem cells from dog ilium and allowing them to differentiate to osteoblasts in vitro. The osteoblast- loaded scaffolds were then placed in cavities in the inferior border of dog mandibles. They observed endochondral bone formation in the Scaffold + MSC group, which seemed to be related to the origin of the stem cells. ${ }^{(22)}$ In the present study, although the newly formed bone was more compact in the the Scaffold+MSC group, it was formed by intramembranous ossification, similar to Jafarian's study.

Because of limitations in financial resources and laboratory facilities, the number of samples in this study was small. The results would have been more generalizable if more dogs had been included in our analysis. Because of the difficulty of radiology in animal study due to general anesthesia, radiological images were not investigated in this study. Further research is required to identify-suitable scaffolds for loading mesenchymal stem cells. In order to determine the role of these cells in healing and tissue regeneration, it might be useful to mark these cells.

\section{Conclusion}

HA/TCP scaffolds and Bio-Oss displayed less bone formation than HA/TCP + MSCs and HA/ TCP + MSCs may induce more bone formation than HA/TCP scaffolds and Bio-Oss.

\section{REFERENCES}

1. Bartee BK. Extraction site reconstruction for alveolar ridge preservation. J Oral Implantol 2001;27:187-97.

2. Pelo S, Boniello R, Gasparini G, Longobardi G, Amoroso PF. Horizontal and vertical ridge augmentation for implant placement in the aesthetic zone. Int J Oral Maxillofac Surg 2007;36:944-8.

3. Wang HL, Al-Shammari K. HVC ridge deficiency classification: A therapeutically oriented classification. Int $\mathrm{J}$ Periodontics Restorative Dent 2002;22:335-43.

4. Hollinger JO, Winn S, Bonadio J. Options for tissue engineering to address challenges of the aging skeleton. Tissue Eng 2000;6:341-50.
5. Barker D, Walls AW, Meechan JG. Ridge augmentation using mandibular tori. Case report. Br Dent J 2001;190: 474-6.

6. Dado DV, Izqueirdo R. Absorption of onlay bone grafts in immature rabbits: membranous versus enchondral bone and bone struts versus paste. Ann Plast Surg 1989;23:3948.

7. Vacanti JP. Beyond transplantation. Arch Surg 1998;123: 545-9.

8. Cooper LF, Harris CT, Bruder SP, Kowalski R, Kadiyala S. Incipient analysis of mesenchymal stem-cell-derived osteogenesis. J Dent Res 2001;80:314-20.

9. Bareille R, Lafage-Proust MH, Faucheux C, Larodhe N, Wenz R, Pard M, Amédée J. Various evaluation techniques of newly formed bone in porous hydroxyapatite loaded with human bone marrow cells implanted in an extra-osseous site. Biomaterials 2000;21:1345-52.

10. Vacanti JP, Morse MA, Saltzman WM, Domb AJ, PerezAtayde A, Langer R. Selective cell transplantation using bioabsorbable artificial polymers as matrices. J Pediatr Surg 1988;23:3-9.

11. Yoshikawa T, Ohgishi H, Tamai S. Immediate bone forming capability of prefabricated osteogenic hydroxyapatite. J Biomed Mater Res 1996;32:481-92.

12. Boo JS, Yamada Y, Okazaki Y, Hibino Y, Okada K, Hata K, Yoshikawa T, Sugiura Y, Uda M. Tissue-engineered bone using mesenchymal stem cells and a biodegradable scaffold. J Craniofac Surg 2002;13:231-43.

13. Jafarian M, Baughman Eslaminejad MB, Khojasteh A, Mashhadi Abbas F, Dehghan MM, Hassanizadeh R, Houshmand B. Marrow-derived mesenchymal stem cellsdirected bone regeneration in the dog mandible: comparison between biphasic calcium phosphate and natural bone mineral. J Oral Surg Oral Med Oral Pathol Oral Radiol Endod 2008;105:e14-24.

14. Ito K, Yamada Y, Nagasaka T, Baba S, Ueda M. Osteogenic potential of injectable tissue-engineered bone: a comparison among autogenous bone, bone substitute (Bio-Oss), platelet-rich plasma and tissue-engineered bone with respect to their mechanical properties and histological findings. J Biomed Mater Res A 2005;73:63-72.

15. Wada K, Niimi, A, Watanabe K, Sawai T, Ueda M. Maxillary sinus floor augmentation in rabbits: a comparative histologic-histomorphometric study between rhBMP2 and autogenous bone. Int $\mathrm{J}$ Periodontics Restorative Dent 2001;21:253-63.

16. Schlegel KA, Fichtner G, Schultze-Mosgau S, Wiltfang J. Histologic findings in sinus augmentation with autogenous bone chips versus a bovine bone substitute. Int $\mathbf{J}$ Oral Maxillofac Implants 2003;18:53-8.

17. Carmagnola D, Adriaens P, Berglundh T. Healing of human extraction sockets filled with Bio-Oss. Clin Oral Implants Res 2003;14:137-43.

18. Shayesteh YS, Khojasteh A, Soleimani M, Alikhasi M, Khoshzaban A, Ahmadbeigi N. Sinus augmentation using 
human mesenchymal stem cells loaded into a beta-tricalcium phosphate/ hydroxyapatite scaffold. Oral Surg Oral Med Oral Pathol Oral Radiol Endod 2008;106:203-9.

19. Niemeyer P, Krause U, Fellenberg J, Kasten P, Seckinger A, Ho AD, Simank HG. Evaluation of mineralized collagen and alpha-tricalcium phosphate as scaffolds for tissue engineering of bone using human mesenchymal stem cells. Cell Tissues Organs 2004:177:68-78.

20. Yang XB, Bhatnagar RS, Li S, Oreffo RO. Biometric collagen scaffolds for human bone cell growth and differenti- ation. Tissue Eng 2004;10:1148-58

21. Weinord C, Gupta R, Huang AY, Weinberg E, Madisch I, Qudsi RA, Neville CM, Pomerantseva I, Vaconti JP. Comparison of hydrogels in the in vitro formation of tissue-engineered bone using mesenchymal stem cells and beta-tricalcium phosphate. Tissue Eng 2007;13:757-65.

22. Wu W, Chen X, Mao T, Chen F, Feng X. Bone marrowderived osteoblasts seeded into porous beta-tricalcium phosphate to repair segmental defect in canine's mandibula. Ulus Travma Acil Cerrahi Derg 2006;12:268-76. 